\title{
Barriers to Vaccination Among People with Parkinson's Disease and Implications for COVID-19
}

\author{
Tiffany Phanhdone ${ }^{\mathrm{a}}$, Patrick Drummond ${ }^{\mathrm{b}}$, Talia Meisel ${ }^{\mathrm{c}}, \mathrm{Naomi}_{\text {Friede }}^{\mathrm{d}}$, Alessandro Di Rocco $^{\mathrm{e}}$, \\ Joshua Chodosh ${ }^{\mathrm{f}, \mathrm{g}}$ and Jori Fleisher ${ }^{\mathrm{a}, *}$ \\ ${ }^{a}$ Section of Movement Disorders, Department of Neurological Sciences, Rush University Medical Center, \\ Chicago, IL, USA \\ ${ }^{\mathrm{b}}$ Marlene and Paolo Fresco Institute for Parkinson's and Movement Disorders, Department of Neurology, \\ New York University School of Medicine, New York, NY, USA \\ ${ }^{\mathrm{c}}$ College of Medicine, SUNY Downstate Health Sciences University, Brooklyn, NY, USA \\ ${ }^{\mathrm{d}}$ Yale School of Nursing, New Haven, CT, USA \\ ${ }^{\mathrm{e}}$ Zucker School of Medicine at Hofstra Northwell, Long Island, NY, USA \\ ${ }^{\mathrm{f}}$ Division of Geriatric Medicine and Palliative Care, Department of Medicine, New York University School of \\ Medicine, New York, NY, USA \\ ${ }^{\mathrm{g}}$ VA New York Harbor Healthcare System, Medicine Service, New York, NY, USA
}

Accepted 31 March 2021

Pre-press 30 April 2021

\begin{abstract}
.
Background: Patients with Parkinson's disease (PD) are at higher risk of vaccine-preventable respiratory infections. However, advanced, homebound individuals may have less access to vaccinations. In light of COVID-19, understanding barriers to vaccination in PD may inform strategies to increase vaccine uptake.

Objective: To identify influenza and pneumococcal vaccination rates, including barriers and facilitators to vaccination, among homebound and ambulatory individuals with PD and related disorders.

Methods: Cross-sectional US-based study among individuals with PD, aged $>65$ years, stratified as homebound or ambulatory. Participants completed semi-structured interviews on vaccination rates and barriers, and healthcare utilization.

Results: Among 143 participants, $9.8 \%$ had missed all influenza vaccinations in the past 5 years, and $32.2 \%$ lacked any pneumococcal vaccination, with no between-group differences. Homebound participants $(n=41)$ reported difficulty traveling to clinic $(p<0.01)$ as a vaccination barrier, and despite similar outpatient visit frequencies, had more frequent emergency department visits $(31.7 \%$ vs. $9.8 \%, p<0.01)$ and hospitalizations $(14.6 \%$ vs. $2.9 \%, p=0.03)$. Vaccine hesitancy was reported in $35 \%$ of participants, vaccine refusal in $19 \%$, and $13.3 \%$ reported unvaccinated household members, with no between-group differences. Nearly $13 \%$ thought providers recommended against vaccines for PD patients, and $31.5 \%$ were unsure of vaccine recommendations in PD.

Conclusion: Among a sample of homebound and ambulatory people with PD, many lack age-appropriate immunizations despite ample healthcare utilization. Many participants were unsure whether healthcare providers recommend vaccinations for people with PD. In light of COVID-19, neurologist reinforcement that vaccinations are indicated, safe, and recommended may be beneficial.
\end{abstract}

\footnotetext{
*Correspondence to: Jori Fleisher, MD MSCE, Rush University Parkinson's Disease and Movement Disorders Program, 1725 W. Harrison Street, \#755, Chicago, IL 60612, USA. Tel.: +1 312563
}

2900; Fax: +1 312563 2024; E-mail: jori_fleisher@rush.edu.; Twitter: @ JoriFleisher 
Keywords: Vaccine hesitancy, vaccination, Parkinson's disease, COVID-19, influenza, pneumonia, health services, homebound

\section{INTRODUCTION}

As the global pandemic of novel coronavirus 19 (COVID-19) enters its second year, over 15 vaccinations are under study, with three granted emergency use authorization in the US, more available abroad, and others awaiting imminent approval $[1,2]$. Despite this, US and European studies already demonstrate hesitancy among the public to accept a COVID-19 vaccination when it becomes available [3-5]. This should not be surprising: in the general US population of adults aged 65 and older, more than one third are not vaccinated for common respiratory pathogens-influenza or pneumococcus - despite widely agreed upon recommendations and full Medicare coverage of their costs [6-8]. To what extent issues of access to care influence such low rates for a Medicare population is not known.

Among those with potential barriers to care, individuals with Parkinson's disease (PD)—comprising $1.3 \%$ of people age 60 years and older, rising to $2.8 \%$ of individuals 90 years and older [9]—may be at higher risk of contracting and suffering complications of respiratory pathogens, with conflicting data on whether this applies to becoming infected with COVID-19 [10-13]. Mounting evidence does suggest that individuals with PD have a higher risk of complications and mortality from COVID-19, and worsening of PD symptoms in the setting of the virus [14]. Over time, individuals with PD grapple with progressive motor impairment and the emergence and progression of nonmotor symptoms [15]. These include dysphagia, which increases aspiration risk, and dementia, psychosis, and depression, which may delay recognition of infectious symptoms $[16,17]$. The increase in the number and severity of these symptoms results in many individuals becoming homebound and surging healthcare utilization in the form of emergency department visits, hospitalizations, and institutionalization [18-21].

Based on our team's experience conducting home visits for individuals with advanced/late-stage PD [22], we hypothesized that individuals who either have lost or are at risk of losing access to neurological care may similarly be at risk of losing access to primary care, and thus, vaccinations [23, 24]. Furthermore, observational data indicate that individuals with moderate and advanced PD are at a 2.5-fold increased risk of hospitalization for influenza and pneumonia, which are both associated with excess morbidity and mortality yet preventable by routine vaccination [25-27]. To our knowledge, only one prior study explored vaccination rates in people with PD. In 1995, Vernon and Jenkins reported that among 29 individuals with PD surveyed, 52\% and 72\%, respectively, had received the influenza and pneumococcal vaccines. No recent studies have explored vaccination rates among people with $\mathrm{PD}$ or whether being homebound had any relationship to these rates. Therefore, we sought to identify influenza and pneumococcal vaccination rates, including barriers and facilitators to vaccination, among both homebound and ambulatory individuals with PD.

\section{MATERIALS AND METHODS}

\section{Standard protocol approvals and consents}

This study was approved by the New York University Grossman School of Medicine Institutional Review Board. All participants provided written or witnessed verbal telephone informed consent.

\section{Study design, setting, participants, and} recruitment

This cross-sectional survey was conducted at the Marlene and Paolo Fresco Institute for Parkinson's and Movement Disorders at New York University Langone Medical Center from September 2016 through May 2017. Study participants were community-dwelling individuals treated at the Fresco Institute, diagnosed with idiopathic PD. Participants were required to be age 65 or older, primarily Englishspeaking, and without significant hearing impairment or speech dysfunction that would preclude participation in a telephone interview. This was an exploratory study funded for one year, with recruitment and enrollment over the span of one influenza season, and thus we based our recruitment goals on enrolling as many homebound participants as possible during that 
time span, and at least double the number of ambulatory participants to allow for comparison. We sought participants through chart review of Fresco Institute patients in the electronic medical record, filtered for those 65 years of age or older with a diagnosis of PD, prioritizing individuals who had opted into research recruitment communication first. They were grouped into either homebound or ambulatory categories by self-report based on questions derived from Medicare homebound criteria [28]. Additionally, we contacted patients who were already enrolled in or on the waiting list for the Edmond J. Safra Interdisciplinary Home Visit Program (HVP), [22] who were known to meet homebound criteria. Participation was voluntary and without incentives.

\section{Survey design}

The survey consisted of standard demographic and disease history questionnaires along with healthcare utilization including: outpatient care by primary care providers and neurologists; the frequency of common colds, influenza, and pneumonia, respectively; self-reported frequency of urgent care, emergency department, and hospital visits; baseline rates of vaccination for influenza and pneumonia; and several questions adapted from the SAGE Working Group on Vaccine Hesitancy [29]. The SAGE questionnaire was revised to appropriately address our study population and age-appropriate vaccinations. We piloted the survey using cognitive interviews with eight participants who were clinically similar to the population studied. After obtaining telephone verbal consent, we conducted a survey lasting approximately 25 minutes and entered responses directly into an electronic database [30].

\section{Measures and outcomes}

We collected demographic information including age, sex, race, ethnicity, primary language, highest level of education, marital status, presence of a care partner, and insurance status. Participants were asked for the year of onset of their PD symptoms, or if unknown, the year of diagnosis, to calculate PD duration. To qualify for homebound status, participants had to indicate that leaving the home requires a considerable effort due to their medical condition, and that leaving requires either an assistive device, special transportation, or the assistance of another individual.

The team asked participants how many times they had received the seasonal influenza vaccine in the past five flu seasons; five years was selected as a more accurate representation of vaccine uptake than a binary response regarding the most recent single flu season. The team also asked how many times participants had ever received a pneumonia vaccine since age 65 . In the latter case, this could include the pneumococcal conjugate vaccine, or PCV13, and the pneumococcal polysaccharide vaccine, or PPSV23. PPSV23 was more recently recommended to include a booster dose, such that participants could have reported a total of $0-3$ pneumonia vaccines. For participants concurrently enrolled in the HVP for clinical care, vaccination records from treating providers were actively sought as part of a quality improvement initiative. The study team gathered the dates of any influenza vaccinations in the preceding five influenza seasons, and the dates of any pneumococcal vaccinations. Vaccination records for non-HVP participants were not verified due to the heterogeneity of care settings in which participants may have received vaccines (i.e., providers within and outside of the institution, free-standing pharmacies, health fairs, etc.). The study team asked whether participants how often they had visited a primary care provider (PCP), neurologist, urgent care center, emergency department, or had been hospitalized, respectively, within the last 12 months. Participants indicated how many times they had experienced a common cold, influenza, and pneumonia, and for each, if the diagnosis was made by a healthcare provider.

The SAGE Core Vaccine Hesitancy Survey assesses beliefs relating to vaccines and healthcare. Participants responded to prompts about whether they believed vaccines were effective, whether others in their household had received all recommended vaccines, if they were ever hesitant about or had refused vaccines themselves, and their reasoning for hesitancy or refusal. Barriers such as distance, time, travel, and cost in obtaining vaccines were specifically solicited. Finally, participants were asked if they thought healthcare providers recommended vaccines for people with PD.

Primary outcome measures were: baseline rates of influenza and pneumococcal vaccination. Secondary outcomes were barriers and facilitators to vaccination uptake as determined by responses to the adapted SAGE Survey. Exploratory outcomes included self-reported illness, healthcare utilization, and correlation between self-reported and verified vaccination status among those with verified vaccination records. 
Table 1

Characteristics of Subjects

\begin{tabular}{|c|c|c|c|c|}
\hline & $\begin{array}{l}\text { Full Cohort } \\
\quad(n=143)\end{array}$ & $\begin{array}{c}\mathrm{HB} \\
(n=41)\end{array}$ & $\begin{array}{c}\text { Ambulatory } \\
(n=102)\end{array}$ & $p$ \\
\hline Age in years, mean & 75.2 & 80.5 & 73.0 & $<0.01$ \\
\hline Female, no. (\%) & $69(48.3)$ & $25(61.0)$ & $44(43.1)$ & 0.05 \\
\hline \multicolumn{5}{|l|}{ Race $^{1}$, no. $(\%)$} \\
\hline White & $132(92.3)$ & $36(87.8)$ & $96(94.1)$ & 0.30 \\
\hline Black & $3(2.1)$ & $2(4.9)$ & $1(1.0)$ & 0.20 \\
\hline Asian & $3(2.1)$ & $1(2.4)$ & $2(2.0)$ & 1.00 \\
\hline Hispanic ethnicity, no. (\%) & $10(7.0)$ & $4(10.0)$ & $6(5.9)$ & 0.39 \\
\hline PD Duration in years, mean (SD) & $9.3(7.0)$ & $12.5(6.4)$ & $8.1(6.8)$ & $<0.01$ \\
\hline Living situation ${ }^{1,2}$, no. $(\%)$ & & & & $\mathbf{0 . 0 3}$ \\
\hline Alone & $39(27.5)$ & $14(35.0)$ & $25(24.5)$ & \\
\hline Spouse/Partner/Significant Other & $91(64.1)$ & $21(52.5)$ & $70(68.6)$ & \\
\hline Other Family Member & $4(2.8)$ & $0(0.0)$ & $4(3.9)$ & \\
\hline Live-in Home Health Aide & $5(3.5)$ & $4(10.0)$ & $1(1.0)$ & \\
\hline Other & $3(2.1)$ & $1(2.5)$ & $2(2.0)$ & \\
\hline Education, no. (\%) & & & & 0.49 \\
\hline$\leq$ High School Diploma & $17(11.9)$ & $7(17.1)$ & $10(9.8)$ & \\
\hline Some College & $14(9.8)$ & $4(9.8)$ & $10(9.8)$ & \\
\hline College Graduate or Higher & $112(78.3)$ & $30(73.1)$ & $82(80.4)$ & \\
\hline \multicolumn{5}{|l|}{ Insurance ${ }^{1}$, no. $(\%)$} \\
\hline Medicare & $107(74.8)$ & $30(73.1)$ & $77(75.5)$ & 0.77 \\
\hline Medicaid & $4(2.8)$ & $3(7.3)$ & $1(1.0)$ & 0.07 \\
\hline Private & $57(39.9)$ & $16(39.0)$ & $41(40.2)$ & 0.90 \\
\hline Self-pay & $1(0.7)$ & $1(2.4)$ & $0(0.0)$ & 0.29 \\
\hline
\end{tabular}

HB, Homebound; PD, Parkinson's disease. Bolded $p$ values indicate statistical significance. ${ }^{1}$ values may summate $>100 \%$ (patients may indicate multiple races or insurance types). ${ }^{2} \mathrm{n}=142$.

\section{Statistical analysis}

Statistical analyses were performed using STATA 15 software (StataCorp LP, College Station, TX). Participants were stratified by homebound status. Continuous variables were assessed for normality, and parametric or non-parametric descriptive statistics were calculated, as appropriate. Categorical variables between homebound and ambulatory participants were summarized by frequencies and percentages using Pearson chi squared, Fisher exact, and two-sample $T$ tests. In the subset of homebound HVP participants for whom vaccination records were available, mean or median differences in self-reported vs. verified vaccinations were calculated, along with a Pearson correlation coefficient $(r)$ for each vaccination type. A two-tailed $p$ value $<0.05$ was considered significant.

\section{RESULTS}

We consented and enrolled 143 individuals, 41 meeting homebound criteria and 102 who were ambulatory. Demographic characteristics are described in Table 1 . The homebound cohort was notably older (80.5 years vs. 73.0 years, $p=<0.01$ ) and reported longer disease duration (12.5 years vs. 8.1 years, $p \leq 0.01)$. Homebound participants were more likely to live alone ( $35 \%$ vs. $24.5 \%, p=0.03$ ). We found no differences between groups in race, ethnicity, education, or insurance status.

Table 2 shows the results for vaccination rates, healthcare utilization, and self-reported illnesses. In the past 5 years, $9.8 \%$ of all participants had missed all influenza vaccinations. There were no significant differences between groups, with $85.4 \%$ of homebound individuals and $92.2 \%$ of ambulatory individuals, respectively, reporting at least one influenza vaccination in the past 5 years. Within the entire cohort, only $67.8 \%$ of participants reported having received any of the age-appropriate pneumococcal vaccinations recommended. Again, we found no differences between groups, with $31.7 \%$ of homebound and $32.3 \%$ of ambulatory individuals, respectively, missing both vaccinations.

Vaccination records were obtained and verified from 20/41 homebound participants. Among those with documented records, homebound individuals with PD had a mean of 3.7 verified influenza vaccinations in the past 5 influenza seasons (SD 1.9, range $0-5)$. The number of vaccinations was over-reported compared with verification in $75 \%$ of this subset: $40 \%$ 
Table 2

Vaccination, Healthcare Utilization, and Self-Reported Illness among Homebound and Ambulatory Individuals with Parkinson's Disease

\begin{tabular}{|c|c|c|c|c|}
\hline & $\begin{array}{l}\text { Full Cohort } \\
(n=143)\end{array}$ & $\begin{array}{l}\text { Homebound } \\
\quad(n=41)\end{array}$ & $\begin{array}{l}\text { Ambulatory } \\
(n=102)\end{array}$ & $p$ \\
\hline Participants with $\geq 1$ Flu Vaccination in Past 5 Years, no. (\%) & $129(90.2)$ & $35(85.4)$ & $94(92.2)$ & 0.23 \\
\hline Flu vaccinations in past 5 years, mean $(\mathrm{SD})$ & $3.9(1.7)$ & $3.9(1.8)$ & $3.9(1.7)$ & 0.97 \\
\hline Participants with > 1 Pneumococcal Vaccination no. (\%) & $97(67.8)$ & $28(68.3)$ & $69(67.7)$ & 0.65 \\
\hline Visits to Primary Care Physician in Past Year, mean (SD) & $3.1(2.3)$ & $3.4(2.6)$ & $2.9(2.1)$ & 0.20 \\
\hline Visits to Neurologist in Past Year, mean (SD) & $3.2(2.0)$ & $3.0(1.8)$ & $3.3(2.1)$ & 0.34 \\
\hline \multicolumn{5}{|l|}{ Subjects With $>2$ Visits in Past Year, no. (\%) } \\
\hline To Urgent Care Clinic & $14(9.8)$ & $7(17.1)$ & $7(6.9)$ & 0.20 \\
\hline To Emergency Department & $23(16.1)$ & $13(31.7)$ & $10(9.8)$ & $<0.01$ \\
\hline To Inpatient Hospital & $9(6.3)$ & $6(14.6)$ & $3(2.9)$ & $\mathbf{0 . 0 3}$ \\
\hline \multicolumn{5}{|l|}{ Self-Reported Illness Frequency, mean (SD) } \\
\hline Colds in Past 2 Years & $1.4(1.3)$ & $1.3(1.5)$ & $1.5(1.2)$ & 0.45 \\
\hline Influenza in Past 5 Years & $0.3(0.8)$ & $0.3(0.7)$ & $0.4(0.8)$ & 0.56 \\
\hline Pneumonia in Past 5 Years & $0.1(0.3)$ & $0.1(0.4)$ & $0.1(0.3)$ & 0.36 \\
\hline
\end{tabular}

Bolded $p$ values indicate statistical significance. $\mathrm{SD}$, standard deviation.

reported 4 more vaccinations than verified; $15 \%$ each reported 3 and 2 more vaccinations than verified, respectively, and 5\% reported 1 more vaccination than verified. Only two individuals had concordance between self-report and verification. For pneumococcal vaccinations, the same 20 homebound individuals had a mean of 0.6 out of 3 possible pneumococcal vaccinations verified. Correlations between self-report and verified vaccination status were weak for both influenza $(r: 0.12)$ and pneumococcal vaccinations (r: 0.43).

In the past year, the entire cohort reported a mean of 3.1 PCP visits, with homebound participants reporting slightly more frequent visits, though this did not approach statistical significance (3.4 vs. 2.9, $p=0.20$ ). In the past year, homebound participants were far more likely to have multiple emergency department visits $(31.7 \%$ of homebound vs. 9.8\% of ambulatory, $p<0.01)$ and multiple hospitalizations ( $14.6 \%$ of homebound vs. $2.9 \%$ of ambulatory, $p=0.03$ ). There were no differences in self-reported illnesses.

We list the SAGE questionnaire responses in Table 3. Overall, 3.5\% of the full cohort did not believe that "vaccines protect people from serious disease" and $10.5 \%$ expressed doubt. Thirty-five percent of the full cohort reported ever being hesitant about a vaccine, $19 \%$ had refused a vaccine, and when asked about vaccination status of household members $13.3 \%$ indicated that their household members were not fully vaccinated, and $37.1 \%$ did not know, with no between-group differences. When asked whether "healthcare providers recommend vaccines for people with PD?" $12.6 \%$ of the entire cohort responded "No", and $31.5 \%$ responded "I don't know". Homebound participants were more likely to indicate that providers recommended against vaccines rather than ambivalence (homebound: $22.0 \%$ "No" and 22.0\% "I don't know", vs. ambulatory: $8.8 \%$ "No" and 35.3\% "I don't know"), however this did not meet statistical significance $(p=0.06)$.

The SAGE questionnaire lists several potential logistical barriers to vaccination, including distance, time, and difficulty traveling to clinic, and cost of the vaccination. Whereas most participants denied these as barriers to vaccination, $12.2 \%$ of homebound participants cited difficulty traveling to clinic as a barrier compared with $1.0 \%$ of ambulatory $(p<0.01)$.

\section{DISCUSSION}

Despite retained access to PCP visits among both ambulatory and homebound individuals with PD, nearly $10 \%$ of individuals in this cohort had missed all influenza vaccinations in the preceding five years, and over $32 \%$ had missed all pneumococcal vaccinations. Besides their own missed vaccinations, participants frequently indicated that their household members were unvaccinated as well. Difficulty traveling to clinic was cited as a barrier to vaccination among homebound participants, however we found no differences in frequency of outpatient clinic visits. This perceived barrier may be exacerbated during the COVID-19 pandemic as visitor restrictions for healthcare appointments introduce additional challenges to attending in-person visits. Furthermore, homebound individuals with PD were more likely to live alone. In a recent meta-analysis, living alone is strongly associated with lower vaccine uptake [31]. 
Table 3

Barriers to Vaccination Based on Responses to Adapted Vaccine Hesitancy Survey

\begin{tabular}{|c|c|c|c|c|}
\hline SAGE Vaccine Hesitancy Survey Question responses (\%) & $\begin{array}{l}\text { Full cohort } \\
(n=143)\end{array}$ & $\begin{array}{l}\text { Homebound } \\
\quad(n=41)\end{array}$ & $\begin{array}{c}\text { Ambulatory } \\
(n=102)\end{array}$ & $p$ \\
\hline Can vaccines protect people from serious disease? & & & & 0.69 \\
\hline No & $5(3.5)$ & $2(4.9)$ & $3(2.9)$ & \\
\hline I don't know & $15(10.5)$ & $5(12.2)$ & $10(9.8)$ & \\
\hline Have the people who live with you been vaccinated with all the recommended vaccines? & & & & 0.11 \\
\hline No & $19(13.3)$ & $6(14.6)$ & $13(12.8)$ & \\
\hline I don't know & $53(37.1)$ & $20(48.8)$ & $33(32.4)$ & \\
\hline \multicolumn{5}{|l|}{ Have you ever been hesitant about a vaccine? } \\
\hline Yes & $35(24.5)$ & $6(14.6)$ & $29(28.4)$ & 0.08 \\
\hline \multicolumn{5}{|l|}{ Have you ever refused a vaccine? } \\
\hline Yes & $19(13.3)$ & $4(9.8)$ & $15(14.7)$ & 0.59 \\
\hline \multicolumn{5}{|l|}{ Are there any reasons you think people should not be vaccinated? } \\
\hline Yes & $46(32.2)$ & $8(19.5)$ & $38(37.3)$ & 0.06 \\
\hline \multicolumn{5}{|l|}{ Are there any reasons you think people with $P D$ should not be vaccinated? } \\
\hline Yes & $7(4.9)$ & $3(7.3)$ & $4(3.9)$ & 0.46 \\
\hline Do healthcare providers recommend vaccines for people with PD? & & & & 0.06 \\
\hline No & $18(12.6)$ & $9(22.0)$ & $9(8.8)$ & \\
\hline I don't know & $45(31.5)$ & $9(22.0)$ & $36(35.3)$ & \\
\hline \multicolumn{5}{|l|}{ Perceived barriers to vaccination } \\
\hline Distance to clinic & $2(1.4)$ & $2(4.9)$ & $0(0.0)$ & 0.08 \\
\hline Time needed to get to clinic & $3(2.1)$ & $0(0.0)$ & $3(2.9)$ & 0.56 \\
\hline Difficulty traveling to clinic & $6(4.2)$ & $5(12.2)$ & $1(1.0)$ & $<0.01$ \\
\hline Cost & $4(2.8$ & $1(2.4)$ & $3(2.9)$ & 1.00 \\
\hline
\end{tabular}

Bolded $p$-values indicate statistical significance.

We also report on two previously undescribed and potentially modifiable barriers to vaccination. While $19 \%$ of all participants reported refusing a vaccine, $31.5 \%$ did not know whether their physicians recommended vaccines for people with PD, and nearly $13 \%$ believed that providers recommended against it. Additionally, over $13 \%$ of participants indicated that their household members were not fully vaccinated, and an additional $37 \%$ were unsure. These discrepancies are actionable opportunities for change. While neurologists are not typically responsible for administering routine vaccinations, this does not preclude recommending or prescribing them. Yet our patients may interpret our silence on vaccines as a tacit contraindication. Numerous interventions have been studied to increase vaccination uptake among older adults, with varying success [32-34]. Posters in clinics or pre-populated reminders in after-visit documentation may communicate neurologists' concurrence with recommendations and encourage care partners to seek their own vaccinations, with minimal administrative burden on providers or staff.

In this study, we found a higher frequency of emergency department and hospital visits among homebound individuals, and no differences in outpatient visits. This challenged our hypotheses that homebound patients would have limited access to routine healthcare, and that this would be an explanation for low vaccination rates. Notably, participants reported visit frequencies without detail on visit content (i.e., sick visits vs. routine follow-up). Analyses of national datasets show over US \$6.7 billion in total excess direct medical costs for outpatient care and physician visits in adults with PD compared with age-matched controls, suggesting that routine care provision is far outstripped by PD-related costs, and discussion of vaccinations may be deferred or missed [35].

The COVID-19 pandemic disproportionately affects older adults [36]. Similar to influenza and pneumonia, individuals with PD-particularly the most advanced and homebound - are at an increased risk of complications from COVID-19 [10-12]. As the rollout of COVID-19 vaccinations continues, attention must be paid to ensuring uptake among high-risk communities, including people with PD. As observed in this and prior studies, homebound individuals with PD are more likely to have advanced disease and live alone [37], which may delay recognition of early signs of infection, leading to more complex, emergent presentations [38, 39]. It is therefore imperative to recognize the importance of age-appropriate vaccinations for this vulnerable population as a modifiable contributor to morbidity and mortality. Prior work has demonstrated increased vaccination uptake through home visits to older adults [32, 40, 41], suggesting 
that such models for individuals with PD [42] may provide an opportunity to advocate for vaccination. Our study suggests that targeted education and interventions may be necessary to address and overcome concerns about COVID-19 vaccines and logistical barriers for individuals with PD. Effective communication strategies between the neurologist, the person with PD, and their household members may reduce vaccine hesitancy and increase uptake, lowering morbidity and mortality [43].

We acknowledge several limitations. First, the study is US-based, and vaccination guidelines, healthcare access, utilization, and socioeconomic status of participants must be interpreted within that healthcare system. Our findings may not apply elsewhere, particularly in single-payer or less developed healthcare systems. Participants were recruited from a single institution in New York City and non-English-speakers were excluded, limiting the generalizability of our findings. Additionally, medical history and vaccination status for the vast majority of participants were obtained by self-report due to logistical and funding constraints, and thus subject to recall and social desirability biases. The lack of vaccination records is a significant limitation and makes it challenging to interpret the low correlation between self-reported and verified vaccinations in the subset for whom records were available. In other populations, the concordance of verified vs. reported vaccinations exceeds $95 \%$, however further work is necessary to assess this in older and cognitively impaired populations [44, 45]. To maximize representation of homebound individuals, we assessed capacity to consent but did not otherwise exclude participants based on cognitive impairment. Particularly in the more advanced homebound PD population, a higher rate of cognitive impairment is anticipated, introducing potential recall bias. Future work should incorporate documented vaccination and healthcare utilization records, and query a broader, more representative population. Interventions to promote vaccine uptake in both ambulatory and homebound PD populations are necessary and underway.

In summary, ambulatory and homebound individuals with PD may be missing routine, age-appropriate vaccinations despite a higher risk of morbidity and mortality from acute respiratory illnesses. One actionable barrier may be a lack of explicit support for vaccination from the patient's treating neurologist. With the rollout of COVID-19 vaccines, now is the time to advocate for preventive care and improve vaccine trust and uptake in our patients and their care partners.

\section{ACKNOWLEDGMENTS}

This work was supported by the Feldstein Medical Foundation, Doris Duke Charitable Foundation Fund to Retain Clinical Scientists, and the Edmond J. Safra Philanthropic Foundation.

N. Friede is supported by Nurse Corps Scholarship grant from the U.S. Department of Health and Human Services

J. Chodosh is supported by 1P30AG066512-01, 1R24AG063725, 1U54AG063546-01, 4R33AG057 291-02, UG3AT009844-01, IIR 17-068, 1R01AG 054574, R01NR016461-01, and CDC BOLD PH $\mathrm{COE}$, receives research support from NIH/NIA, NIH/NINR, the New York State Department of Health, and SCAN Health Plan, Long Beach CA, and is on the editorial board of the Journal of the American Geriatrics Society.

J. Fleisher is supported by K23NS097615 and U01NS100610, receives research support from NIH/NINDS, NIA/Emory Roybal Center for Dementia Caregiving Mastery, PCORI, and Parkinson's Foundation, serves on the editorial board of AAN Brain \& Life magazine, receives royalties from UpToDate, and serves as a consultant for UCB Biosciences, Inc.

\section{CONFLICT OF INTEREST}

The authors have no conflicts of interest to report.

\section{REFERENCES}

[1] Al-Kassmy J, Pedersen J, Kobinger G (2020) Vaccine candidates against coronavirus infections. Where does COVID-19 stand? Viruses 12.

[2] Kaur SP, Gupta V (2020) COVID-19 Vaccine: A comprehensive status report. Virus Res 288, 198114.

[3] Fisher KA, Bloomstone SJ, Walder J, Crawford S, Fouayzi H, Mazor KM (2020) Attitudes toward a potential SARSCoV-2 vaccine: A survey of U.S. adults. Ann Intern Med 173, 964-973.

[4] Dror AA, Eisenbach N, Taiber S, Morozov NG, Mizrachi M, Zigron A, Srouji S, Sela E (2020) Vaccine hesitancy: The next challenge in the fight against COVID-19. Eur $J$ Epidemiol 35, 775-779.

[5] COCONEL Group (2020) A future vaccination campaign against COVID-19 at risk of vaccine hesitancy and politicisation. Lancet Infect Dis 20, 769-770.

[6] Gnanasekaran G, Biedenbender R, Davidson HE, Gravenstein S (2016) Vaccinations for the older adult. Clin Geriatr Med 32, 609-625. 
[7] Peck M, Gacic-Dobo M, Diallo MS, Nedelec Y, Sodha SV, Wallace AS (2019) Global Routine Vaccination Coverage, 2018. MMWR Morb Mortal Wkly Rep 68, 937-942.

[8] Bridges CB, Hurley LP, Williams WW, Ramakrishnan A, Dean AK, Groom AV (2015) Meeting the challenges of immunizing adults. Vaccine 33(Suppl 4), D114-20.

[9] Savica R, Grossardt BR, Rocca WA, Bower JH (2018) Parkinson disease with and without Dementia: A prevalence study and future projections. Mov Disord 33, 537-543.

[10] Del Prete E, Francesconi A, Palermo G, Mazzucchi S, Frosini D, Morganti R, Coleschi P, Raglione LM, Vanni P, Ramat S, Novelli A, Napolitano A, Battisti C, Giuntini M, Rossi C, Menichetti C, Ulivelli M, De Franco V, Rossi S, Bonuccelli U, Ceravolo R, Tuscany Parkinson COVID-19 Participants (2021) Prevalence and impact of COVID-19 in Parkinson's disease: Evidence from a multi-center survey in Tuscany region. $J$ Neurol 268, 1179-1187.

[11] Brown EG, Chahine LM, Goldman SM, Korell M, Mann E, Kinel DR, Arnedo V, Marek KL, Tanner CM (2020) The effect of the COVID-19 pandemic on people with Parkinson's disease. J Parkinsons Dis 10, 1365-1377.

[12] van der Heide A, Meinders MJ, Bloem BR, Helmich RC (2020) The impact of the COVID-19 pandemic on psychological distress, physical activity, and symptom severity in Parkinson's disease. J Parkinsons Dis 10, 13551364.

[13] Fasano A, Cereda E, Barichella M, Cassani E, Ferri V, Zecchinelli AL, Pezzoli G (2020) COVID-19 in Parkinson's disease patients living in Lombardy, Italy. Mov Disord 35, 1089-1093.

[14] Bloem BR, Trenkwalder C, Sanchez-Ferro A, Kalia LV, Alcalay R, Chiang H-L, Kang UJ, Goetz C, Brundin P, Papa SM (2021) COVID-19 vaccination for persons with Parkinson's disease: Light at the end of the tunnel? J Parkinsons Dis 11, 3-8.

[15] Kalia LV, Lang AE (2015) Parkinson's disease. Lancet 386, 896-912.

[16] Schrag A, Hommel AL a. J, Lorenzl S, Meissner WG, Odin P, Coelho M, Bloem BR, Dodel R, CLaSP consortium (2020) The late stage of Parkinson's -results of a large multinational study on motor and non-motor complications. Parkinsonism Relat Disord 75, 91-96.

[17] Titova N, Qamar MA, Chaudhuri KR (2017) The nonmotor features of Parkinson's disease. Int Rev Neurobiol 132, 33-54.

[18] Hassan A, Wu SS, Schmidt P, Dai Y, Simuni T, Giladi N, Bloem BR, Malaty IA, Okun MS, Investigators N-Q (2013) High rates and the risk factors for emergency room visits and hospitalization in Parkinson's disease. Parkinsonism Relat Disord 19, 949-54.

[19] Safarpour D, Thibault DP, DeSanto CL, Boyd CM, Dorsey ER, Racette BA, Willis AW (2015) Nursing home and endof-life care in Parkinson disease. Neurology 85, 413-419.

[20] Hosking A, Hommel AALJ, Lorenzl S, Coelho M, Ferreira JJ, Meissner WG, Odin P, Bloem BR, Dodel R, Schrag A, Care of Late Stage Parkinsonism (CLaSP) Consortium (2021) Characteristics of patients with late-stage parkinsonism who are nursing home residents compared with those living at home. J Am Med Dir Assoc 22, 440-445.e2.

[21] Fabbri M, Kauppila LA, Ferreira JJ, Rascol O (2020) Challenges and perspectives in the management of late-stage Parkinson's disease. J Parkinsons Dis 10, S75-S83.

[22] Fleisher J, Barbosa W, Sweeney MM, Oyler SE, Lemen AC, Fazl A, Ko M, Meisel T, Friede N, Dacpano G, Gilbert RM, Rocco AD, Chodosh J (2018) Interdisciplinary home visits for individuals with advanced Parkinson's disease and related disorders. J Am Geriatr Soc 66, 1226-1232.

[23] Bloem BR, Henderson EJ, Dorsey ER, Okun MS, Okubadejo N, Chan P, Andrejack J, Darweesh SKL, Munneke M (2020) Integrated and patient-centred management of Parkinson's disease: A network model for reshaping chronic neurological care. Lancet Neurol 19, 623-634.

[24] Lin CC, Hill CE, Burke JF, Kerber KA, Hartley SE, Callaghan BC, Skolarus LE (2021) Primary care providers perform more neurologic visits than neurologists among Medicare beneficiaries. J Eval Clin Pract 27, 223-227.

[25] Pilishvili T, Bennett NM (2015) Pneumococcal disease prevention among adults: Strategies for the use of pneumococcal vaccines. Am J Prev Med 49, S383-90.

[26] Black CL, Williams WW, Warnock R, Pilishvili T, Kim D, Kelman JA (2017) Pneumococcal vaccination among medicare beneficiaries occurring after the advisory committee on immunization practices recommendation for routine use of 13 -valent pneumococcal conjugate vaccine and 23 -valent pneumococcal polysaccharide vaccine for adults aged $\geq 65$ years. MMWR Morb Mortal Wkly Rep 66, 728-733.

[27] Leibson CL, Maraganore DM, Bower JH, Ransom JE, O'Brien P C, Rocca WA (2006) Comorbid conditions associated with Parkinson's disease: A population-based study. Mov Disord 21, 446-55.

[28] (2014) Pub 100-02 Medicare Benefit Policy: Clarification of the Confined to the Home Definition in Chapter 15, Covered Medical and Other Health Services, of the Medicare Benefit Policy Manual.

[29] SAGE Working Group on Vaccine Hesitancy (2014) Report of the SAGE Working Group on Vaccine Hesitancy.

[30] Harris PA, Taylor R, Thielke R, Payne J, Gonzalez N, Conde JG (2009) Research electronic data capture (REDCap)a metadata-driven methodology and workflow process for providing translational research informatics support. $J$ Biomed Inf 42, 377-81.

[31] Jain A, van Hoek AJ, Boccia D, Thomas SL (2017) Lower vaccine uptake amongst older individuals living alone: A systematic review and meta-analysis of social determinants of vaccine uptake. Vaccine $\mathbf{3 5}, \mathbf{2 3 1 5 - 2 3 2 8 .}$

[32] Thomas RE, Lorenzetti DL (2018) Interventions to increase influenza vaccination rates of those 60 years and older in the community. Cochrane Database Syst Rev 5, CD005188.

[33] Wateska AR, Nowalk MP, Lin CJ, Harrison LH, Schaffner W, Zimmerman RK, Smith KJ (2019) An intervention to improve pneumococcal vaccination uptake in high risk 5064 year olds vs. expanded age-based recommendations: An exploratory cost-effectiveness analysis. Hum Vaccines Immunother 15, 863-872.

[34] Betsch C, Rossmann C, Pletz MW, Vollmar HC, Freytag A, Wichmann O, Hanke R, Hanke W, Heinemeier D, Schmid P, Eitze S, Weber W, Reinhardt A, Küpke NK, Forstner C, Fleischmann-Struzek C, Mikolajetz A, Römhild J, Neufeind J, Rieck T, Suchecka K, Reinhart K (2018) Increasing influenza and pneumococcal vaccine uptake in the elderly: Study protocol for the multi-methods prospective intervention study Vaccination60. BMC Public Health $\mathbf{1 8}, 885$.

[35] Yang W, Hamilton JL, Kopil C, Beck JC, Tanner CM, Albin RL, Ray Dorsey E, Dahodwala N, Cintina I, Hogan P, Thompson T (2020) Current and projected future economic burden of Parkinson's disease in the U.S. NPJ Parkinsons Dis 6, 15.

[36] Shahid Z, Kalayanamitra R, McClafferty B, Kepko D, Ramgobin D, Patel R, Aggarwal CS, Vunnam R, Sahu N, Bhatt 
D, Jones K, Golamari R, Jain R (2020) COVID-19 and older adults: What we know. J Am Geriatr Soc 68, 926-929.

[37] Nwabuobi L, Barbosa W, Sweeney M, Oyler S, Meisel T, Di Rocco A, Chodosh J, Fleisher JE (2019) Sex-related differences in homebound advanced Parkinson's disease patients. Clin Interv Aging 14, 1371-1377.

[38] Qiu WQ, Dean M, Liu T, George L, Gann M, Cohen J, Bruce ML (2010) Physical and mental health of homebound older adults: An overlooked population. J Am Geriatr Soc 58, 2423-2428.

[39] Xiang X, Chen J, Kim M (2020) Trajectories of homebound status in medicare beneficiaries aged 65 and older. Gerontologist 60, 101-111.

[40] Dalby DM, Sellors JW, Fraser FD, Fraser C, van Ineveld C, Howard M (2000) Effect of preventive home visits by a nurse on the outcomes of frail elderly people in the community: A randomized controlled trial. CMAJ 162, 497-500.
[41] Fabacher D, Josephson K, Pietruszka F, Linderborn K, Morley JE, Rubenstein LZ (1994) An in-home preventive assessment program for independent older adults: A randomized controlled trial. J Am Geriatr Soc 42, 630-638.

[42] Fleisher JE, Sweeney MM, Oyler S, Meisel T, Friede N, Di Rocco A, Chodosh J (2019) Disease severity and quality of life in homebound people with advanced Parkinson disease. Neurol Clin Pract 10, 277-286.

[43] Harrison EA, Wu JW (2020) Vaccine confidence in the time of COVID-19. Eur J Epidemiol 35, 325-330.

[44] Llupià A, García-Basteiro AL, Mena G, Ríos J, Puig J, Bayas JM, Trilla A (2012) Vaccination behaviour influences self-report of influenza vaccination status: A cross-sectional study among health care workers. PloS One 7, e39496.

[45] King JP, McLean HQ, Belongia EA (2018) Validation of self-reported influenza vaccination in the current and prior season. Influenza Other Respir Viruses 12, 808-813. 